\title{
An Elementary Derivation of The Black Hole En- tropy in Any Dimension
}

\section{Carlos Castro}

Center for Theoretical Studies of Physical Systems, Clark Atlanta University, Atlanta, GA. 30314. E-mail: castro@ctsps.cau.edu

Received: 30 April 2000 / Accepted: 22 November 2000 / Published: 26 March 2001

\begin{abstract}
An elementary derivation of the Black Hole Entropy area relation in any dimension is provided based on the New Extended Scale Relativity Principle and Shannon's Information Entropy. The well known entropy-area linear Bekenstein-Hawking relation is derived. We discuss briefly how to derive the most recently obtained Logarithmic and higher order corrections to the linear entropy-area law in full agreement with the standard results in the literature.
\end{abstract}

Keywords: Entropy, New Relativity, Clifford Oscillator; p-branes, Black Holes.

(c)2001 by the author. Reproduction for noncommercial purposes permitted. 


\section{Introduction: The New Relativity Theory}

We deem it important to review the New Extended Scale Relativity and its postulates. This is all one needs to derive the generalized entropy-area relation in just a few steps.

Recently we have proposed that a New Relativity principle may be operating in Nature which could reveal important clues to find the origins of $M$ theory [1]. We were forced to introduce this new Relativity principle, where all dimensions and signatures of spacetime are on the same footing, to find a fully covariant formulation of the $p$-brane Quantum Mechanical Loop Wave equations. This New Relativity Principle, or the principle of Polydimensional Covariance as has been called by Pezzaglia, has also been crucial in the derivation of Papapetrou's equations of motion of a spinning particle in curved spaces that was a long standing problem which lasted almost 50 years [2]. A Clifford calculus was used where all the equations were written in terms of Clifford-valued multivector quantities; i.e one had to abandon the use of vectors and tensors and replace them by Clifford-algebra valued quantities, matrices, for example.

The New Extended Scale Relativity Theory has already allow us to derive, from first principles, the String Uncertainty Relations, corrections thereof, and the precise connection between the Regge trajectory behaviour of the string spectrum and the area quantization. The full blown infinite-dimensional Quantum Spacetime Generalized Uncertainty relations that included the contributions of all $p$-branes, not only due to strings, was given [3]. In [4] we were able to show that there are no such things as EPR paradoxes in this New Scale Relativity. The latter is truly a Machian Relativity theory, were relationships are the only meaningful statements one can make. Originally it was formulated in an abstract Categorical space, C-spaces [1]. The New Relativity sprang out from Laurent Nottale's Scale Relativity [5]; Mohammed El Naschie Transfinite Cantorian-Fractal Spacetime [6]; Garnet Ord's original work on fractal random walks [7]; the classic Geoffrey Chew's Bootstrap hypothesis: all p-branes are made of each other (William Pezzaglia's principle of polydimensional covariance); and $p$-adic Physics and Non-Archimedean Geometry by Siddarth, Pitkannen, Khrennikov, Freund, Volovich, Vladimorov, Zelenov and many others [8].

In this short letter we will derive the Black-Hole Area-Entropy Relation, in any dimension, in a couple of straightforward steps, based on the New Relativity and Shannon's information theory. We will discuss briefly the most recently obtained Logarithmic corrections to the linear law based on a $p$-loop harmonic oscilator living in Clifford manifolds [20, 21]. In general, the area-entropy relation has a clear departure from linearity [22]. The goals and partial list of sucesses of the New Relativity, formulated in Cantorian-Fractal Spacetime, is:

1. Provide with a truly background independent formulation of Quantum Gravity and $M$ theory. 
2. To furnish the physical foundations underlying String and $M$ theory. So far nobody has been able to answer the question: "What is String Theory?". The New Relativity may provide with a plausible answer. In particular we were able to derive the string uncertainty relations directly from the wave equations of the New Relativity [10] and its $p$-brane generalizations.

3. Prove why we live in $3+1$ dimensions. In [9] we have shwon that te average dimension of the world is of the order of $4+\phi^{3}$.

4. Solve the cosmological constant problem [9], in a similar fashion that Nottale did in his initial work on Scale Relativity. And discuss of the existence of a dimensional phase transition from $4+\phi^{3}$ to $\phi^{3}$, the so called Noncommutative quasi-crystal phase.

5. Provide with a physical meaning to negative topological dimensions and to the notion of negative-information entropy, or anti-entropy as has been called by M. Conrad.

6. Write down the Unique Quantum Master Action functional of the world, in an abstract Cspace, outside spacetime, a generalized Twistor space, that governs the quantum dynamics for the creation of spacetime itself; gravity and all of the other fundamental forces in Nature.

7. Propose a solution to how chiral symmetry-breaking occurs in Nature and much, much more $[10]$.

With this preamble let us summarize briefly what are the basic postulates of the New Relativity Theory next. This will allow us to explain in full rigour why the fundamental constants in Nature, like Planck's constant, change with "time"; i.e. with the Renormalization Group flow of the microscopic (scaling) arrow of time: with energy or resolutions. We will explain why there is an effective value of the Planck constant when one approaches scales comparable with the Planck length: when quantum gravitational phenomena are important.

\subsection{The Postulates of the New Scale Relativity Theory}

1. Physics is an experimental science. Physics is about measurements. In order to perform a measurement one needs a standard of measure to compare measurements with. In Einstein's Relativity he introduced the speed of light as the velocity standard to compare velocities with. From the mathematical point of view one can say: let us have a field at $\mathbf{x}$. To define the real number $\mathbf{x}$, mathematically, requires knowing its value with infinite precision: simply adding digits at will. To do it physically is another story. It would require a computer an inifinite amount of 
memory just to store all this infinite amount of information. One way to avoid the problem of using real numbers is to introduce $p$-Adic numbes in Physics [8].

For these reasons, Laurent Nottale [5] introduced his original Scale Relativity by postulating that the Planck scale is the universal standard of measurements. It is the minimum, impassible distance in Nature, invariant under scale-relativistic dilatations. Exactly along similar lines, Einstein's motion Relativity was based on taking the speed of light as the maximum, and invariant, attainable speed in Nature. The Planck scale in four dimensions is given in terms of the 3 fundamental constants, speed of light $c$, Newton's constant in four dimensions $G$ and Planck's constant $\hbar$ :

$$
\Lambda=\sqrt{\frac{\hbar G}{c^{3}}}=10^{-33} \mathrm{cms} .
$$

Notice that if $\hbar=0, G=0, c=\infty$ the Planck scale would have been automatically zero. Meaning that one would not have "quantum mechanics"; the gravitational force will reduce to 0 and there would not be "Lorentz invariance", only Galilean symmetry. The invariant minimum scale in all dimensions, $\Lambda$ will be set to unity; in units where $\hbar=c=1$. The Planck scale is explictly dimension dependent through its dependence on the Newton constants. So is the Newton gravitational constant. For example, in $D, D-1, D-2, \ldots$ the Planck scale (that we set to unity) is given in terms of the Newton constants:

$$
\Lambda=G_{D}^{(1 / D-2)}=G_{D-1}^{(1 / D-3)}=\ldots=1 .
$$

Taking logarithms (in any base if one wishes) on eq-(2a), one has the relationship among the Newton constants, in $D$ and $D-1$ diemnsions, which does not require any compactifications whatsoever, as it is assumed in conventioanl string and Kaluza-Klein supergravities:

$$
\frac{D-3}{D-2}=\frac{\ln G_{D-1}}{\ln G_{D}}=\frac{\log G_{D-1}}{\log G_{D}} .
$$

Notice that when $D=2$, the Newton constant in two-dimensions is set to $G_{2}=1$ so that $1^{\infty}=1$. For example in $D=2, G_{2}=1 \rightarrow \ln G_{2}=\ln 1=0$ which is consistent with all the denominators of eq- $(2 \mathrm{~b})$.

Variable speed of light Cosmologies are becoming very popular today. This does not imply that Einstein was wrong at his time. This only means, as Dirac pointed out long ago, that the fundamental constants can change slowly with "time": the value of the constants slowly flow with the Renormalization group, from the Ultraviolet (small scales) to the Infrared (large scales). The speed of light when Einstein formulated his theory is the same as today. Only during the early Universe there were substantial changes. The constants flow with the provision that the truly fundamental "relativistic" invariant in Nature, the Planck scale, remains fixed. Eq-(1) by 
inspection, entails that $\hbar, c, G$ could flow with the RG flow in such a fashion that they will leave $\Lambda$ invariant. This explains how, at very large energies (Planckian), at very small scales (Planck scales), we can begin to see the corrections to the fundamental constants. We have now an understanding as to why one has an effective Planck constant that can vary with energy once we approach scales comparable to the Planck scale. Exactly the same thing happens when we approach the speed of light: the masses begin to grow compared to their rest mass values.

2. The Principle of Poly-dimensional covariance and the Clifford-algebra-multivector calculus.

The New Relativity is a true Machian one. Relationships are the only meaningful statements one can make. To view a single $p$-brane as an isolated entity is a meaningless concept. $p$-branes are solely defined in terms of others. For this reason, we included the Chew boostrap hypothesis as a crucial ingredient, and wrote:

All $p$-branes are made of each-other. This is, in essence, the origins of the dualities in $M$ theory. Pezzaglia [2] using Chew's bootstrap hypothesis coined the term "poly-dimensional-covariance". Since now we have all $p$-branes, of all dimensionalities from $p=0,1,2, \ldots, \infty$, the poly-dimensional covariance is the statement that all $p$-branes (dimensions) rotate into each-other. Exactly what happens with ordinary Lorentz transformations: the axis are entangled and space and time are mixed. For the role of objects of negative topological dimensions see [10]. For example, in ordinary string theory, an object of dimension $p=-1$ spans a $p+1=-1+1=0$-dimensional "worldline": it is an instanton.

Space and time have different units. Einstein was able to connect them by the introduction of an invariant velocity parameter: the speed of light. If one sets $c=1$ in Einstein's theory, space and time have the same "units": they are exchangeable. Since the Planck scale has units of length, upon setting $\Lambda=1$, it means that all dimensions are are exchangeable also. Dimensions then can be "rotated" into each-other. p-branes can be transformed into each-other (duality principle). Einstein's Relativity required the use of a Lorentz four-vector to embrace space with time; energy with momentum, etc. .... In the New Relativity one needs Clifford-algebra-valued multivectors. The latter multi-vector is a mathematical object that encodes all objects of different dimensionalities. It encodes all $p$-brane histories, embedded in a target spacetime background, into one single scoop. For the analog of ordinary Lorentz transformations that rotate space into time, for example, one has polydimensional transformations, that rotate $p$-branes among each-other.

For this reason we will write our New Relativistic wave equations in a Clifford-space, whose derivative elements will be quadratic and have, in addition to the ordinary quadratic derivatives with respect to ordinary point coordinates, $x^{\mu}$, quadratic derivatives w.r.t the holographic areacoordinates, holographic volume-coordinates, .... We will explain this in detail in section 2 what these holographic area, volume, hypervolume, ... coordinates really are.

3. Einstein's General Relativity required a Riemannian Geometry; the New Relativity requires 
a Cantorian-Fractal Spacetime model developed by Mohammed El Naschie [6]. The latter is an example of Von Neuman's Noncommutative Geometry. The world is multi-fractal. For a detailed analyis of Noncommutative geometry, etc. ... see [10] and references therein.

Essentially one has that because the Planck scale is the minimum distance in Nature; there are no such things as "points" in Nature. Only in Mathematics. A "point" is smeared into a fuzzy ball of all possible topological dimensision. On average, the "points" are four-dimensional spheres, for this reason we live effectively in four-dimensions: we perceive an average dimension taken over all the infinite-dimensional spacetime [10]. This occurs exactly in the same fashion that we only measure the average velocity of an ensemble of molecules in a room, when we measure the temperature of the room, pressure, etc. ... Cantorian-Fractal Geometry is a "pointless" Noncommutative Geometry. As we zoom into what looks like a "point" from a distance, we realize that it is a four-dimensional sphere. As we zoom in deeper into what we thought looked like a point inside that small sphere we realize that it is a smaller four-dimensional sphere; and so forth ad infinitum. We cannot reach the Planck scale. Like a massive object cannot reach the speed of light. Nature is multifractal.

4. Non-Archimedean geometry and p-Adic Physics [8].

Since the Planck scale is the minimum attainable scale in Nature, Nottale provided with the scaling analogs of Lorentz transformations: The composition of two scalings (contractions) cannot yield scales smaller than the Planck scale. In the same way that the addition of two velocities cannot exceed the speed of light. This forces one to abandon the Archimedean Geometry for a Non-Archimedean one and the replacement of real numbers for $p$-Adic ones [8]. Using a $p$-Adic norm, which allows to define $p$-adic numbers based on another extension of the rational numbers, one can show that the net resulting $p$-adic norm, of the composition/sum of two $p$-adic numbers, is less than the $p$-adic norm of the larger norm of the initial two $p$-adic numbers. Roughly speaking, the $p$-adic norm obeys an ultra-metricity condition. $p$-Adics are the natural numbers to use in the New Relativity. The latter Relativity is consitent with a Non-Archimedean Geometry.

What we find most important in using primes, the atoms of numbers, in the New Relativity is that all prime numbers must appear on equal footing. In the same way that all dimensions did. Since dimensions change this automatically entails that one must include also all topologies on equal footing: a "Topological Relativity" as has been called by Finkelstein and others. $p$ Adic numbers have been used to label topologies. Not surprisingly, this fits very well within this new framework. It is also well known to the experts that there is a very deep connection between Quantum groups and $p$-Adic numbers when the deformation parameter $q=1 / p$. The Real number limit $p=\infty$ is equivalent to the classical group limit $q=0$. Quantum Symmetric spaces "interpolate" between the Real numbers and the $p$-Adic ones.

To sum up, the world is not scale invariant. Dimensions are in the eye of the beholder $[1,2]$. 
They are resolution-dependent [5, 6]. As we probe into smaller regions, larger energies, with the "microscope" of the RG flow, more dimensions are accesible to us. Things look different to two observers living in two different scale-frames of references. The most famous example: it is meaningless to compare the vacuum energies in two completely different scale-frames of references: the Planck scale and the Hubble scale. This is the fundamental reason why the "cosmological" constant differs by 60 orders of magnitude: the so-called cosmological constant "problem". This inconsistency, was elegantly solved by Nottale [5] and recently by us [9] within the context of Renormalization Group techniques and self-organized non-equlibrium critical phenomena in Cosmology, intially emphasized by Smolin and Kauffmann [19].

Based on this cursory introduction to the basic principles of the New Relativity, we can understand now why the Planck constant $\hbar$, for example, is not a true constant, but it varies with energy, resolution. Once, of course, we approach the Planck scale. At ordinary energies, $\hbar$ is a constant. This is why these effects have not been detected in ordinary experiments. The energy is extremely low in comparison with the Planck energy of $10^{19} \mathrm{GeV}$.

\section{Explicit Derivation of the Black Hole Entropy-Area Relations from the New Rel- ativity Theory}

Having gone through a brief tour of the the postulates of the New Relativity, we shall present in this section the simple steps to derive the Black-Hole Entropy-Area linear relation and its logarithmic (and higher order) corrections. leaving all the details to references [20, 21]. We only need to discuss in detail the generalized wave equations (for the $p$-loop oscillator) in a Clifford-manifold that encode the dynamics such family of $p$-brane or $p$-loop harmonic oscillators.

Imagine one has a family of $p$-branes moving in a target spacetime background, where the values of $p$ range from $p=0$, a point history; $p=1$ a closed string history; $p=2$ a closed membrane history. A membrane of topology of a sphere, for example: a 2-loop; and so forth. Until one saturates the spacetime with the spacetime filling $p$-brane: $p+1=D$. The family of $p$-brane degrees of freedom are encoded in term of hyper-matrix coordinates [1].

Generalized "hyper-matrix coordinates" transformations in the New Relativity reshuffle, for example, a loop history into a membrane history; a membrane history into a into a 5-brane history; a 5-brane history into a 9-brane history and so forth; in particular it can transform a $p$-brane history into suitable combinations of other $p$-brane histories as building blocks. This is the bootstrap idea taken from the point particle case to to the $p$-branes case: each brane is made out of all the others. "Lorentz" transformations in C-spaces involve hypermatrix changes of "coordinates" [1]. The naive Lorentz transformations do not apply in the world of Planck scale physics. Only at large scales the Riemannian continuum is recaptured. For a discussion 
of the more fundamental Finsler Geometries implementing the minimum scale (maximal proper acceleration) in String Theory see [13].

There was a one-to-one correspondence between the nested hierarchy of point, loop, 2-loop, 3-loop, ..., p-loop histories encoded in terms of hypermatrices [1] and wave equations written in terms of Clifford-algebra valued multivector quantities [2]. This permitted us to recast the QM wave equations associated with the hierarchy of nested p-loop histories, embedded in a target spacetime of $D$ dimensions, where the values of $p$ range from: $p=0,1,2,3, \ldots, D-1$, as a single QM line functional wave equation whose lines live in a Noncommutative Clifford manifold of $2^{D}$ dimensions. $p=D-1$ is the the maximum value of $p$ that saturates the embedding spacetime dimension.

An action line functional, associated with the interacting QFT of lines in Noncommutative Clifford manifolds C-spaces, was launched forward in [1]. The QFT program of such interacting field theory of $\mathbf{C}$-lines in Noncommutative spaces, a generalized Twistor theory, is currently under investigation [18]. One will have cubic interactions associated with the product and coproduct of a Braided-Hopf-Quantum-Clifford algebra [15]. The product represents the annihilation of two $\mathrm{C}$-lines into a third one. The coproduct represents the creation of two lines from one line. The quartic interactions correspond to the braiding of two-lines into another two-lines: scattering. One has here more complicated statistics than the ordinary bose/fermions one: it is a braided one!. The two-point vertex corresponds to a pairing of the algebra representing the composition of two lines into a 0-line. The kinetic terms are the extensions of Witten-Zwiebach open/closed string field theory, based on the Batalin-Vilkovisky Quantum Master action [16, 17]. The closedstring field theory action required the used of Operads and Gerstenhaber algebras [17]. Such QFT in Noncommutative spaces is a very complex one due to the Ultra-Violet/Infrared entanglement. Due to string duality, there is a maximum scale dual to the minimum Planck scale. In [9] we provided with integral expressions to determine the maximum scale that is dual to the Planck scale.

The line functional wave equation in the Clifford manifold, C-space, for the simplest "linear" case is:

$$
\int d \Sigma\left(\frac{\delta^{2}}{\delta X(\Sigma) \delta X(\Sigma)}+\mathcal{E}^{2}\right) \Psi[X(\Sigma)]=0
$$

where $\Sigma$ is an invariant evolution parameter of $l^{D}$ dimensions generalizing the notion of the invariant proper time in Special Relativity linked to a massive point particle line (path) history:

$$
(d \Sigma)^{2}=\left(d \Omega_{p+1}\right)^{2}+\Lambda^{2 p}\left(d x^{\mu} d x_{\mu}\right)+\Lambda^{2(p-1)}\left(d \sigma^{\mu \nu} d \sigma_{\mu \nu}\right)+\Lambda^{2(p-2)}\left(d \sigma^{\mu \nu \rho} d \sigma_{\mu \nu \rho}\right)+\ldots
$$

$\Lambda$ is the Planck scale in $D$ dimensions: $\Lambda=G_{D}^{\frac{1}{D-2}}$ where $G_{D}$ is Newton's constant in $D$ dimensions. 
$\mathbf{X}(\Sigma)$ is a Clifford-algebra valued "line" living in the Clifford manifold (C-space):

$$
X=\Omega_{p+1}+\Lambda^{p} x_{\mu} \gamma^{\mu}+\Lambda^{p-1} \sigma_{\mu \nu} \gamma^{\mu} \gamma^{\nu}+\Lambda^{p-2} \sigma_{\mu \nu \rho} \gamma^{\mu} \gamma^{\nu} \gamma^{\rho}+\ldots
$$

The multivector $\mathbf{X}$ encodes in one single stroke the point history represented by the ordinary $x_{\mu}$ coordinates and the holographic projections of the nested family of $\mathbf{1}$-loop, 2-loop, 3 -loop, ... p-loop histories onto the embedding coordinate spacetime planes given respectively by:

$$
\sigma_{\mu \nu}, \sigma_{\mu \nu \rho}, \ldots, \sigma_{\mu_{1} \mu_{2} \ldots \mu_{p+1}}
$$

The scalar $\Omega_{p+1}$ is the invariant proper $p+1=D$-volume associated with the motion of the (maximal dimension) $\mathbf{p}$-loop across the $D=p+1$-dim target spacetime. It naturally couples to the unit matrix of the Clifford algebra.

There was a coincidence condition [1] that required to equate the values of the center of mass coordinates $x_{\mu}$, for all the $\mathbf{p}$-loops, with the values of the $x^{\mu}$ coordinates of the point particle path history. This was due to the fact that upon setting $\Lambda=0$ all the $\mathbf{p}$-loop histories collapse to a point history. The latter history is the baseline where one constructs the whole hierarchy. This also required a proportionality relationship:

$$
\tau \sim \frac{A}{\Lambda} \sim \frac{V}{\Lambda^{2}} \sim \ldots \sim \frac{\Omega_{p+1}}{\Lambda^{p}}
$$

$\tau, A, V, \ldots, \Omega^{p+1}$ represent the invariant proper time, proper area, proper volume, .., proper $p+1$-dim volume swept by the point, loop, 2-loop, 3-loop, . ., p-loop histories across their motion through the embedding spacetime, respectively. $\mathcal{E}=T$ is a quantity of dimension $(\text { mass })^{p+1}$, the maximal $p$-brane tension $(p=D-1)$.

A C-line in C-space is nothing but a Clifford algebraic extension of Penrose's twistors. From a distance, the line looks like a point history: a one-dimensional world line. Upon closer inspection, upon zooming in, we realize that it corresponds to the center-of-mass motion of a closed string history, a 1-loop. And that the line turns into a two-dimensional surface: the lateral area-swept by the closed-string. Upon a further inspection, as we zoom in deeper, we realize that the closedstring history is really a closed-membrane history; and so forth and so forth. Dimensions are resolution and energy dependent. All these $p$-brane histories that have a common center-of-mass coordinate, are encoded in terms of the Clifford-algebra-valued $\mathbf{C}$-lines, a generalized twistor. The holographic, or shadow-projections, of the areas, volumes, hypervolumes, ... onto the respective coordinate planes are nothing but the holographic coordinates of the $\mathbf{C}$-lines.

The wave functional $\Psi$ is in general a Clifford-valued, hypercomplex number. In particular it could be a complex, quaternionic or octonionic valued quantity. At the moment we shall not dwell on the very subtle complications and battles associated with the quaternionic/octonionic 
extensions of Quantum Mechanics [14] based on Division algebras and simply take the wave function to be a complex number. The line functional wave equation for lines living in the Clifford manifold (C-spaces) are difficult to solve in general. To obtain the Bekenstein-Hawking BlackHole Entropy-Area relations, and corrections thereof, one needs to simplify them. The most simple expression (all modes are frozen except the zero modes) is to write the simplified wave equation for the $p$-loop harmonic oscillator, in units $\hbar=c=1$ :

$$
\begin{gathered}
\left\{-\frac{1}{2 \Lambda^{p-1}}\left[\frac{\partial^{2}}{\partial x^{\mu} \partial x_{\mu}}+\Lambda^{2} \frac{\partial^{2}}{\partial \sigma^{\mu \nu} \partial \sigma_{\mu \nu}}+\Lambda^{4} \frac{\partial^{2}}{\partial \sigma^{\mu \nu \rho} \partial \sigma_{\mu \nu \rho}}+\ldots\right]+\right. \\
\left.\frac{m_{p+1}}{2 L^{2}}\left[\Lambda^{2 p} x_{\mu}{ }^{2}+\Lambda^{2 p-2} \sigma_{\mu \nu}^{2}+\ldots \Omega_{p+1}^{2}\right]\right\} \Psi=T \Psi\left[x^{\mu}, \sigma^{\mu \nu}, \sigma^{\mu \nu \rho}, \ldots\right] .
\end{gathered}
$$

The solutions of the latter $p$-loop oscillator wave equations where given in $[21,22]$ in terms of the dimensionless (rescaled) variables:

$$
\tilde{x}_{\mu}=\frac{\Lambda^{p} x_{\mu}}{L} . \quad \tilde{\sigma}_{\mu \nu}=\frac{\Lambda^{p-1} \sigma_{\mu \nu}}{L} . \quad \tilde{\Omega}_{p+1}=\frac{\Omega_{p+1}}{L} .
$$

where the analog of oscillator amplitude is $L$ given by:

$$
L^{2}=\frac{\Lambda^{p+1}}{m_{p+1}} \Rightarrow \Lambda^{p+1}<L<\frac{1}{m_{p+1}} .
$$

where $m_{p+1}$ is the analog of the Compton momentum.

$$
\Psi \sim \exp \left[-\left(\tilde{x}_{\mu}^{2}+\tilde{\sigma}_{\mu \nu}^{2}+\ldots\right)\right] H_{n_{i}}\left(\tilde{x}_{\mu}\right) H_{n_{j k}}\left(\tilde{\sigma}_{\mu \nu}\right) H_{n_{j k l}}\left(\tilde{\sigma}_{\mu \nu \rho}\right) \ldots
$$

we are expressing as usual the ground state as the Gaussian and the excited ones by the product of the Hermite polynomials. The excitations of the $p$-loop oscillator are collective ones given by the set of center of mass excitations; holographic area, holographic volume, ... excitations:

$$
N=\left\{n_{i} ; n_{i j} ; n_{i j k}, \ldots\right\} .
$$

The Tension is quantized as follows [21, 22]:

$$
T_{N}=\left(N+\frac{1}{2} 2^{D}\right) m_{p+1}
$$

the degree of the Clifford algebra in $D$ dimesnions ("number of bits") is $2^{D}$. The first collective excited state corresponds to setting all the quantum numbers equal to 1 in full compliance with the principle of dimensional democracy ( $p$-brane democracy) or poly-dimensional covariance: all dimensions must appear on the same footing: 


$$
\left\{n_{i}=1 ; n_{i j}=1 ; n_{i j k}=1, \ldots\right\} \Rightarrow N_{1}=2^{D}
$$

The degeneracy of the the $\mathcal{N}^{t h}=k$ state is given as a function of $D, N$ :

$$
d g\left(D, N_{k}\right)=\frac{\Gamma\left(2^{D}+N_{k}\right)}{\Gamma\left(N_{k}+1\right) \Gamma\left(2^{D}\right)} . \quad N_{k}=k 2^{D} . \quad k=1,2,3, \ldots
$$

The degeneracy of the first collective excited state is $N_{1}=2^{D}$ and is naturally given by simply setting $N=2^{D}$ in the above equation:

$$
d g\left(N=2^{D}\right)=\frac{\Gamma(2 N)}{\Gamma(N+1) \Gamma(N)}
$$

The Entropy is defined as the natural logarithm of the degeneracy. Hence taking the logarithm and using Stirling's asymptotic expansion of the logarithms of the Gamma functions yields for the first collective excited state the following Entropy:

$$
\text { Entropy }=S=2 N \ln (2)-\frac{1}{2} \ln (N)-\frac{1}{2} \ln (4 \pi)-O(1 / N) .
$$

Before invoking Shannon's information entropy by setting the number of holographic $p$-loop bits to coincide precisely with the ratio of the $d$-2-dimensional area associated with a black hole horizon in $d$-dimensions $(d \neq D)$ in the following manner: $N \sim A / G$, one needs to justify this assumption. Most importantly is to answer the following questions:

Where does Einstein's gravity come from? How is it obtained as the long distance effective theory from the "gas" of highly-excited $p$-loop oscillators quanta associated with New Relativty in C-spaces (Clifford manifolds)?

- At the moment we cannot answer such difficult questions; however we recall that the low energy limit of string theory (the effective string action after integrating out the massive string modes) reproduces Einstein-Hilbert action with the ordinary scalar curvature term plus a series of higher powers of the curvature. Einstein's gravity is recovered as the low energy limit from strings propagating in curved backgrounds.

- Fujikawa [23] has given strong reasons why Shannon's Information Entropy is related to the Quantum Statistical (Thermodynamical) Entropy. In particular in understanding the meaning of Temperature.

- Li and Yoneya [24], among many others, have derived the Bekentein-Hawking entropy-area linear relation by taking the logarithm of the degeneracy of the highly excited massive (super)string states in $d$-dimensions. 
- The New Relativity principle advocates that dimensions are in the eye of the beholder $[1,2]$. In one reference frame an observer sees a gas of $p$-loop oscillators in $D$-dimensions in the first collective excited state of $N=2^{D}$. In another frame of reference another observer sees only strings in $d$ dimensions $(d \neq D)$ in a very highly excited state $n$.

If we identify Shannon's information entropy as the number of bits $N=2^{D}$ of the $p$-loop oscillator which is just the degree of the Clifford algebra in $D$ dimensions, Shannon's information entropy can them be re-expressed in terms of the number of bits as follows:

$$
N=S_{\text {Shannon }}=\log _{2}\left(2^{N}\right)=N=2^{D} \Rightarrow \text { Number of states }=\mathcal{N}=2^{N}=2^{2^{D}} .
$$

Notice the double exponents defining the number of states. The Black-Hole Horizon literarily is an information horizon as well!

Now we are ready to make our only assumption. We will identify the number of $p$-loop bits $N=2^{D}$ in $D$ dimensions to coincide precisely with the number of area bits contained by a BlackHole horizon (in $d$ dimensions) of a given Area in units of the Planck scale. Namely it is given by the ratio of the areas for the radius $R$ and $\Lambda$ respectively:

$$
N=2^{D}=\frac{A_{d-2}(R)}{A_{d-2}(\Lambda)} \sim \frac{A_{d-2}}{G}=\frac{A_{d-2}}{\Lambda^{d-2}} . \quad G_{d}=\Lambda^{d-2}
$$

where we just wrote down the value of the Newton constant $G_{d}$ in $d$-dimensions. The number of transverse dimensions to the radial $r$ and temporal coordinates $t$ of a spherically-symmetric black hole is $d-2$. So the Horizon area refers to a $(d-2)$-dimensional one.

This is all we need to obtain precisely the Black-Hole entropy-area relation in the literature including the logarithmic corrections [22], up to numerical coefficients, directly from eq-(16):

$$
S \sim[2 \ln (2)](A / G)-\frac{1}{2} \ln (A / G)-\frac{1}{2} \ln (4 \pi)-O(1 /(A / G))+\ldots
$$

To conclude: We have obtained in a very straightforward fashion not only the BekensteinHawking entropy-area linear relation for a Black Hole in any dimensions but also the logarithmic corrections plus higher order corrections [21, 22]. All of these corrections appear with a minus sign and the entropy-area relation satisfies the law of black hole thermodynamics:

$$
\text { If } A_{3}>A_{1}+A_{2} \Rightarrow S\left(A_{3}\right)-S\left(A_{1}\right)-S\left(A_{2}\right)>0 \text {. }
$$

If two black holes of areas $A_{1}, A_{2}$ merge to give another black hole of area $A_{3}>A_{1}+A_{2}$ then the resulting entropy cannot decrease. For an upper bound on the values of $A_{3}$ see [21]. In Planck units we obtained:

$$
\frac{A_{2} A_{2}}{G^{2}}>\frac{A_{3}}{G}>\frac{A_{1}+A_{2}}{G}
$$


For further details of all the technicalities behind this construction we refer to [21, 22]. If one wishes to obtain the relation among the quantities $R, \Lambda, D, d$ one simply may use the definition (18) of the number of bits:

$$
N=2^{D}=\left(\frac{R}{\Lambda}\right)^{d-2} \Rightarrow D \log _{2}=(d-2) \log _{2}(R / \Lambda) .
$$

which means that if $R=\Lambda \rightarrow d=\infty$ which agress with Nottale's Scale Relativity. Upon reaching the impassible Planck scale, the (fractal) dimension blows up since it takes an infinite energy to probe Planck scale resolutions. Therefore, by excitations one means those relative to the Planck scale. Setting $N_{1}=N_{2}=1$ will violate the second law of Black Hole Thermodynamics since $1+1=2>1.1$. Hence one needs at least $N_{1}=N_{2}=2$ so that $2+2=4=2.2$.

\section{Acknowledgements}

We thank E.Spallucci, S.Ansoldi, D. Finkelstein, A.Granik, S. Duplij, L. Nottale, M.S. El Naschie, D, Chakalov, M. Pitkannen, W. Pezzaglia, for many discussions. 


\section{References}

[1] 1-Castro, C. "Hints of a New Relativity Principle from p-brane Quantum Mechanics" hepth/9912113.

Journal of Chaos, Solitons and Fractals 2000, vol. 11 (11), 1721.

Castro, C. "The Search for the Origins of $M$ Theory: Loop QM and Bulk/Boundary Duality" hep-th/9809102.

Ansoldi, S; Castro, C; Spallucci, E. Class. Quant. Gravity 1999 vol. 16, 1833. hepth/9809182.

[2] Pezzaglia, W. "Dimensionally Democratic Calculus and Principles of Polydimensional Physics" gr-qc/9912025.

[3] Castro, C. "Is Quantum Spacetime Infinite Dimensional?" hep-th/0001134. Journal of Chaos, Solitons and Fractals 2000, vol. 11 (11), 1663.

[4] Castro, C; Granik, A. "How the New Scale Relativity resolves some Quantum Paradoxes" Journal of Chaos, Solitons and Fractals 2000, vol. 11 (11), 2167.

[5] Nottale, L. Fractal Spacetime and Microphysics, Towards the Theory of Scale Relativity; World Scientific 1992.

Nottale, L. La Relativite dans Tous ses Etats; Hachette Literature. Paris. 1999.

[6] El Naschie, M.S. Jour. Chaos, Solitons and Fractals 1999, vol 10 nos. (2-3), 567.

[7] Ord, G. Jour. Chaos, Solitons and Fractals 1999, vol 10 nos. (2-3), 499.

[8] Pitkannen, M. "p-Adic TGD: Mathematical Ideas" hep-th/9506097.

Khrennikov, A. "p-Adic numbers in Classical and QM ..." quant-ph/0003016.

Altaisky, M; Sidharth, B. Jour. Chaos, Solitons and Fractals 1999, vol. 10 (2-3), 167.

Vladimorov, V; Volovich, I; Zelenov, E. p-Adic Numbers in Mathematical Physics; World Scientific, Singapore 1994.

Brekke, L; Freund, P. Physics Reports 1993, vol. 231, 1-66.

Ghoshal, D; Sen, A. "Tachyon Condensation and Brane Descent Relations in p-Adic String Theory" hep-th/0003278.

[9] Castro, C; Granik, A; El Naschie, M.S "Why we live in 3+ 1 Dimensions" hep-th/0004152. 
[10] Castro, C. Foundations of Physics Letts. 1997, vol. 10, 273.

Castro, C "the String Uncertainty Relations follow from the New Relativity Principle" Foundations of Physics 2000, vol. 30 (8), 1301-1317.

Castro, C. Journal of Chaos, Solitons and Fractals 2000 vol. 11 (11), 1663.

[11] Connes, A. Noncommutative Geometry. Academic Press. New York. 1994.

[12] Majid, S. Foundations of Quantum Group Theory; Cambridge University Press. 1995.

Majid, S. Int. Jour. Mod. Phys. 2000, A 5, 4689.

Biedenharn, L.C.; Lohe, M.A. Quantum Groups and q-Tensor Algebras; World Scientific. Singapore, 1995.

[13] 13- Brandt, H. Jour. Chaos, Solitons and Fractals 1999, vol. 10 (2-3), 267.

[14] Adler, S. Quaternionic Quantum Mechanics and Quantum Fields; Oxford Univ. Press, New York. 1995.

[15] Osiewicz, Z. "Clifford-Hopf Algebra and Bi-Universal Hopf Algebra" q-alg/9709016.

[16] Witten, E. Nuc. Phys. B. 1986, 268, 253.

[17] Zwiebach, Z. Nuc. Phys. B. 1993, 390, 33.

[18] Ansoldi, S; Aurilia, A; Castro, C; Spallucci, E. "Quenched-Minisuperspace Bosonic p-Brane Propagator" Univ. of Trieste (2000) preprint.

[19] Smolin, L; Kaufmann, S "Combinatorial Dynamics in Quantum Gravity" hep-th/9809161.

[20] Castro, C. "The Status and Programs of the New Relativity Theory" physics/0011040.

Castro, C; Granik, A. "Derivation of the Logarithmic Corrections to the Black-Hole Entropy from the New Relativity".

[21] Castro, C; Granik, A. "P-loop Oscillator on Clifford manifolds and Black Hole Entropy" physics/0008222 v2.

[22] Majumdar, P. "Quantum Aspects of Black Hole Entropy" hep-th/0009008.

[23] Fujikawa, K. "Shannon's Statistical Entropy and the H-Theorem in Quantum Statistical Mechanics" cond-mat/0005496

[24] Li, M; Yoneya, T. Journal of Chaos, Solitons and Fractals 1999 vol. 10 (2-3), 429. 